\title{
The role of radiology in the diagnosis and treatment of mesenteric ischaemia
}

\author{
Sara Upponi, ${ }^{1}$ John Julian Harvey, ${ }^{2,3}$ Raman Uberoi, ${ }^{4}$ Arul Ganeshan ${ }^{3}$
}

${ }^{1}$ Department of Radiology, Addenbrooke's Hospital, Cambridge, UK

${ }^{2}$ Royal Centre for Defence Medicine, Queen Elizabeth Hospital, Birmingham, UK

${ }^{3}$ Department of Interventional Radiology, Birmingham Heartlands Hospital, The Heart of England NHS Trust, Birmingham, UK

${ }^{4}$ Department of Interventional Radiology, John Radcliffe Hospital NHS Trust, Oxford, UK

\section{Correspondence to}

Dr Arul Ganeshan, Department of Interventional Radiology, The Heart of England NHS Trust, Bordesley Green East, Birmingham B9 5SS, UK; aganeshan@hotmail.com

Received 15 April 2012 Revised 1 October 2012 Accepted 15 November 2012

Published Online First

14 December 2012

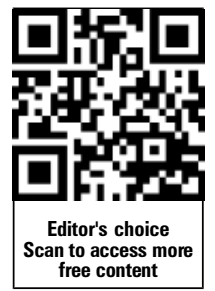

To cite: Upponi $\mathrm{S}$, Harvey JJ, Uberoi R, et al. Postgrad Med J

2013;89:165-172.

\section{ABSTRACT}

Clinicians working in any acute medical/surgical unit need an understanding of mesenteric ischaemia. Acute mesenteric ischaemia is a life-threatening vascular emergency associated with high morbidity and mortality. However, prompt diagnosis with the use of contrastenhanced CT, more specifically CT angiography, has replaced catheter angiography as the new standard and is readily available in many emergency departments. Similarly, new hybrid open surgery endovascular treatment can minimise the surgical insult to these often critically ill elderly patients. Together, these changes can change the previously grim prognosis associated with this condition.

By contrast, chronic mesenteric ischaemia (CMI) is an insidious disease and often a diagnosis of exclusion. However, it can cause a significant reduction in a patient's quality of life, due to 'mesenteric angina' and food avoidance, yet can potentially be treated simply and effectively. Recognition of the typical clinical history and imaging findings is key to making the diagnosis in a timely fashion.

Radiology plays a significant role in the diagnosis and increasingly in the treatment of mesenteric ischaemia. Other clinicians should have a basic understanding of what radiology can and cannot offer. The advantages and limitations of commonly used imaging modalitiesplain films, CT, MRI and ultrasound, are examined. The significance of findings, such as pneumatosis coli and portal gas are explained. Finally, the different endovascular management of both acute and $\mathrm{CMI}$ is discussed, which have emerged as minimally invasive options to complement open revascularisation surgery.

\section{INTRODUCTION}

Mesenteric ischaemia is an acute or chronic perfusion abnormality of the gastrointestinal tract. Acute mesenteric ischaemia (AMI) is a life-threatening vascular emergency, with a mortality of up to $80 \% .{ }^{1}$ However, there has been a slight improvement in recent years, perhaps due to wider use of contrast-enhanced CT allowing earlier definitive diagnosis and better control of atrial fibrillation, reducing systemic thromboembolism, one of the major underlying causes. ${ }^{2}$ Chronic mesenteric ischaemia (CMI) is a more insidious disease, which can cause severe cachexia and result in a significant reduction in patient quality of life. ${ }^{3}$ Around 20\% of patients with CMI go on to develop AMI, offering a high-risk population in whom earlier diagnosis and treatment could potentially reduce the catastrophic impact of $\mathrm{AMI}^{4}{ }^{4}$ Unfortunately however, many patients' first presentation is with bowel infarction, posing the question of how these patients can be identified earlier.
The diagnosis of mesenteric ischaemia is clinically difficult, due to the non-specific symptoms and signs. However, AMI is an important diagnosis to consider typically in elderly patients with acute abdominal pain out of proportion to clinical signs, particularly those with a previous history of vascular disease or atrial fibrillation. CMI is a diagnosis of exclusion, but a history of postprandial pain and weight loss is suggestive, highlighting the importance of taking a careful patient history. Early diagnosis is important to improve patient symptoms and to prevent bowel infarction with its associated morbidity and mortality.

This article reviews the relevant causes and vascular anatomy needed to understand mesenteric ischaemia. Radiology plays an increasingly important role in the diagnosis and treatment of both AMI and CMI. The key imaging findings on various different modalities are discussed. Endovascular treatment provides an attractive and increasingly popular option. The role of endovascular treatments is outlined.

\section{EPIDEMIOLOGY}

The true incidence of mesenteric ischaemia is difficult to quantify. Around $20 \%$ of patients $>65$ years old in the general population, and an even higher percentage of patients with peripheral vascular disease have significant atherosclerotic changes in the mesenteric vasculature. ${ }^{3} 5$ However, most of these patients are asymptomatic and do not seek medical attention. Conversely, AMI is uncommon, accounting for one in 1000 hospital admissions. ${ }^{6}$ However, it is more common than ruptured abdominal aortic aneurysms. ${ }^{2}$

Most patients with mesenteric ischaemia are elderly ( $>65$ years) and have multiple comorbidities, such as chronic obstructive pulmonary disease, previous stroke and peptic ulcer disease, which can complicate the diagnosis and treatment. ${ }^{278}$ These comorbidiities and lack of physiological reserve may partly explain the high morbidity and mortality rates, but also underscores the need to maintain a high suspicion of mesenteric ischaemia in this patient group.

Female patients account for the majority of patients with AMI, but this appears to be due to the relative higher frequency of women living into old age. $^{2}$

The commonest cause of AMI is acute superior mesenteric artery (SMA) occlusion (67\%) with mesenteric vein thrombosis, and non-occlusive ischaemia (low flow states) accounting for roughly $15 \%$ each. $^{2}$ Venous thrombosis tends to occur in younger patients (mean age 50 years) and has the best prognosis. ${ }^{2}$ Non-occlusive disease has the 
worst prognosis reflecting the poor underlying clinical condition in these patients, who are often critically ill from other causes. $^{2} 7$

\section{MESENTERIC VASCULAR ANATOMY}

Knowledge of the relevant vascular anatomy is key to understanding and diagnosing mesenteric ischaemia. Similarly, a sound knowledge of the normal and vascular pattern and common anatomical variants is essential for assessing angiograms and planning endovascular treatment. ${ }^{9}$

The mesenteric viscera are supplied by three main arteries, the coeliac artery (CA), the SMA and inferior mesenteric artery (IMA). These originate sequentially from the anterior surface of the abdominal aorta around the levels of T12, L1 and L3/4 vertebral bodies, respectively. This can be useful for rapid vessel identification and reducing the patient's intravenous contrast load during endovascular treatment. ${ }^{10}$

The CA supplies the foregut (oesophagus to second part of the duodenum). The SMA supplies the mid-gut (second part of duodenum to the distal two-thirds of the transverse colon). The IMA supplies the hindgut (distal one-third of the transverse colon to the proximal rectum). The SMA has a more acute angle of origin with abdominal aorta compared with the other mesenteric arteries, making it more susceptible to thromboemboli. ${ }^{2}$ Similarly, this acute angle must be remembered when considering endovascular treatment to ensure appropriate catheter selection, or if a brachial approach is used. ${ }^{10}$

The SMA arises just distal to the CA and descends into the right iliac fossa where it terminates as the ileocolic artery. The SMA gives origin to multiple jejunal and ileal branches, as well as the right and middle colic arteries. It may also give rise to the hepatic artery or accessory hepatic arteries. Importantly, collateral pathways exist between the mesenteric arteries allowing potential cross-perfusion (by retrograde flow) in case of the vessel occlusion. A variety of other collateral vessels can develop and are commonly seen with CMI, although these collaterals take time to form. ${ }^{3}$ The CA and SMA are linked via the gastroduodenal and pancreaticoduodenal arteries. The marginal artery of Drummond and arc of Riolan provide collaterals between SMA and IMA territories. ${ }^{9}$ Further collaterals from the IMA are provided by rectal collaterals supplied via the internal iliac arteries (figure 1).

Venous drainage of the small bowel and colon is via the superior and inferior mesenteric veins. The inferior mesenteric vein drains into the splenic vein, which unites with the superior mesenteric vein (SMV) to form the portal vein. The portal vein is easily identified on CT imaging and can be traced retrogradely to identify the contributory veins.

The ileum is the most commonly infarcted segment of bowel, followed by the jejunum and then the colon. This reflects the watershed regions of the bowel and overlap with other arterial networks. Around $50 \%$ of patients may have infarction to all three parts of the bowel. ${ }^{8}$

\section{AETIOLOGY OF MESENTERIC ISCHAEMIA}

The aetiology of mesenteric ischaemia can be divided into occlusive arterial, venous thrombosis and non-occlusive causes. This is shown in table 1 . In the majority of cases, the underlying cause is endoluminal. External compression syndromes, for instance, median arcuate ligament syndrome or compression by an abdominal mass lesion are less common. ${ }^{3}$

The commonest cause of acute arterial occlusion is embolic, closely followed by thrombosis. ${ }^{2}$ Thrombi occur more proximally in the artery and, therefore, are associated with more severe

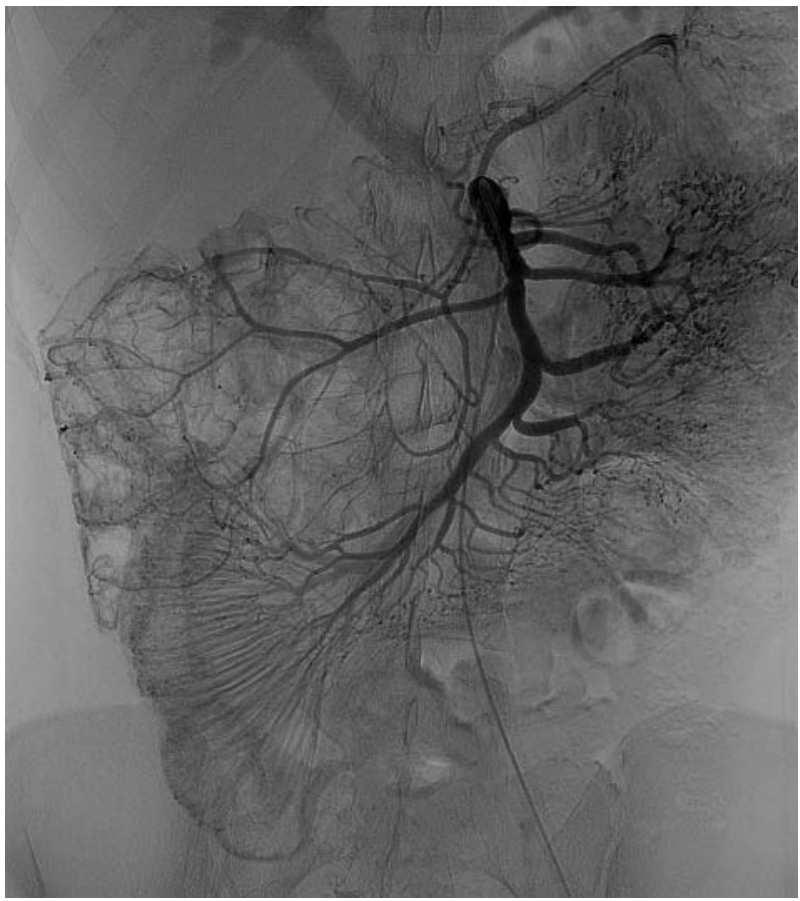

Figure 1 Catheter angiography of the superior mesenteric artery depicting jejunal and ileal vascular arcades.

intestinal infarction. ${ }^{8}$ Thrombi tend to occur in patients with a background history of malignancy or other prothrombotic states, whereas, emboli occur in patients with previous myocardial infarction and atrial fibrillation. Most thromboemboli (predominantly of cardiac origin) are sufficiently small to lodge distal to the middle colic artery, classically sparing the proximal jejunum and ascending colon. Atherosclerotic emboli (eg, from unstable plaques) are smaller still, causing short-segment bowel ischaemia depending on which branch vessels are affected. ${ }^{4}$

Cardiac thrombi and synchronous emboli to other vascular territories, for instance, the renal arteries, are found commonly. Consequently, the heart should be evaluated for thrombus as a potential source of emboli, as well as reviewing other viscera for evidence of ischaemia. ${ }^{4}{ }^{8}$ As with all acute occlusive vascular diseases, time is a critical factor in preventing end organ damage. Diagnosis and treatment within $24 \mathrm{~h}$, and early use of contrast-enhanced CT, have been shown to contribute to a reduction in mortality. ${ }^{8}$

Table 1 Causes of acute and chronic mesenteric ischaemia

\begin{tabular}{cl}
\hline Acute & \\
Occlusive arterial & Thromboembolic disease \\
& Dissection \\
& Transection (iatrogenic/trauma) \\
& Extrinsic compression (eg, median arcuate ligament) \\
Occlusive venous & Thrombophilia and hypercoagulable states (eg, malginancy) \\
& Extrinsic compression \\
Non-occlusive & Hypotension \\
& Extrinsic mesenteric compression (hernias, adhesions) \\
& Drugs (ergot derivatives, cocaine use) \\
Chronic & Atherosclerotic disease \\
& Arterial hyperplasia \\
& Vasculitides \\
\hline
\end{tabular}




\section{CLINICAL PRESENTATION OF MESENTERIC ISCHAEMIA}

At rest, the small bowel and colon receive $10-15 \%$ of the cardiac output, rising to up to $35 \%$ postprandially. ${ }^{1}$ Most of the blood supply is to the mucosa, which is therefore at risk early during mesenteric ischaemia. Vascular compromise causes pain, malabsorption and sloughing, seen as haemorrhage. ${ }^{4}$ Progression of ischaemia results in loss of peristalsis, progressive mural oedema, dilatation and eventually perforation, as subsequent layers of the bowel wall are affected. Progression depends on whether there is reperfusion or further deterioration due to progressive or continuing occlusion. ${ }^{7}$

The presentation of AMI is very different to CMI. AMI causes severe abdominal pain, which is often accompanied by nausea, vomiting and bloody diarrhoea. ${ }^{4}{ }^{6}$ Classically with AMI, the patient's pain is out of proportion to the clinical findings, and the diagnosis should be considered in all elderly patients presenting with severe abdominal pain, particularly in those with atrial fibrillation or known vascular disease. These patients are often critically unwell, and there may be signs of sepsis and peritonism if bowel infarction or perforation has occurred. Clinical findings may be supported by a raised lactate and neutrophilia, although these are non-specific and late findings. ${ }^{1} \quad 4$ A very elevated d-dimer level $(>1.5 \mathrm{mg} / \mathrm{ml}$, normal range $<0.3 \mathrm{mg} / \mathrm{ml}$ ) has been shown to be a strong, but not specific risk factor for AMI, while a normal d-dimer excludes AMI. ${ }^{11}$ Increased age, bleeding per rectum and an insidious onset of symptoms were shown to be the greatest risk factors for poor outcome in a retrospective review of 163 Swedish patients with confirmed AMI. ${ }^{12}$

CMI is insidious in onset due to worsening chronic mesenteric atherosclerotic disease, which allows development of collateral vessels. ${ }^{3}$ Mesenteric atherosclerosis is common in the elderly population, and it is unclear why only a proportion of this patient group develops symptoms of CMI. The classical history is of pain occurring $0-3 \mathrm{~h}$ after meals, so-called 'abdominal angina' or 'mesenteric angina'. ${ }^{3}$ This can lead to food avoidance and severe cachexia over a period of several months to years. ${ }^{3}$ This highlights the importance of elucidating a clear history, to differentiate this weight loss from occult malignancy. Invariably, these patients will undergo cross-sectional imaging to exclude other causes for their symptoms, potentially allowing $\mathrm{CMI}$ to be diagnosed and treated before it progresses to AMI.

\section{THE ROLE OF DIAGNOSTIC IMAGING IN MESENTERIC ISCHAEMIA \\ Abdominal x-ray}

The utility of the plain abdominal image has been debated and is performed less commonly in the emergency department for acute abdominal pain. The diagnostic yield for AMI is low and generally non-specific. Around 25\% of patients with AMI have a normal abdominal x-ray (AXR), consequently, a normal AXR does not exclude the diagnosis. ${ }^{1}$ However, certain signs should be recognised on AXR, which may support the diagnosis when interpreted in conjunction with an appropriate patient history.

Ischaemia disrupts peristalsis leading to ileus. Bowel wall oedema may be visible as 'thumb printing', although this is seen in $<40 \%$ of AMI. ${ }^{1}$ As ischaemia progresses to infarction, there is increasing gaseous dilatation and then thinning of the bowel wall. Pneumatosis coli may be visualised as locules of gas or linear lucencies within the bowel wall (figure 2). Portal venous gas may be visible as linear branching lucencies within the periphery of the liver or in the region of the small-bowel mesentery, and is associated with a poor prognosis. ${ }^{1}$ Bowel perforation will

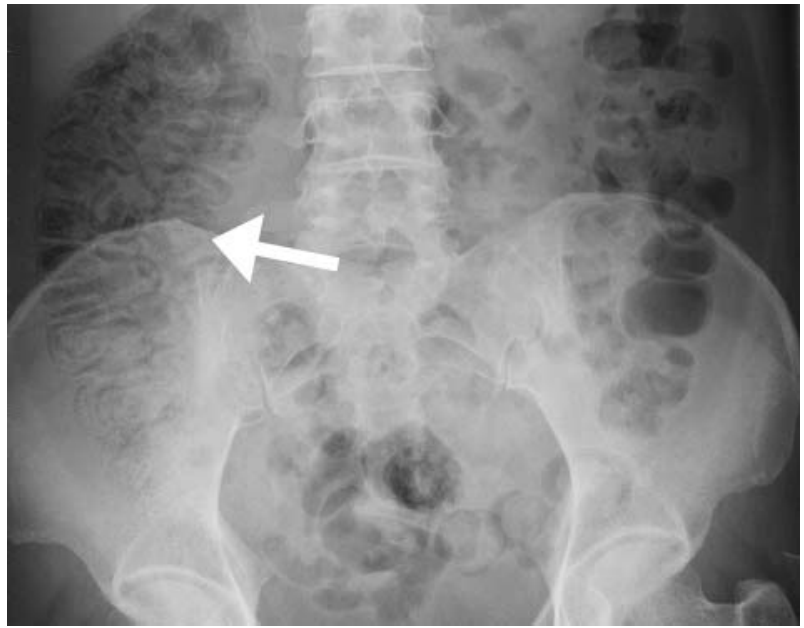

Figure 2 Supine abdominal plain film demonstrating linear pneumatosis of the caecum and ascending colon (arrow).

lead to free subphrenic gas, which may be useful in stratifying the patient for emergency laparotomy. Vascular calcification indicates background atherosclerotic disease, which although common, may be a clue to the diagnosis. ${ }^{11}$

\section{Ultrasound}

Ultrasound has little role in the diagnosis of AMI. ${ }^{4}{ }^{6}$ The proximal arteries only can be visualised even in skilled hands, and this is often challenging due to overlying bowel gas (eg, ileus) or patient body habitus. The finding of a proximal vessel stenosis/ occlusion may be misleading on its own as this is a common finding in the asymptomatic elderly population. This is likely due to established collateral pathways which are not identified at ultrasound. ${ }^{6}$ Intraoperative ultrasound may be utilised in assessing the response of treatment and bowel viability. ${ }^{4}$

Ultrasound may have a role in the non-acute presentation, for instance, in identifying portal vein or SMV thrombus. ${ }^{13}$ Similarly, Doppler spectral analysis of the peak systolic velocity in the proximal CA and SMA can give an estimation of vessel stenosis and patency, which may be useful in stratifying patient risk. ${ }^{3}$

\section{Multidetector CT}

Contrast-enhanced multidetector CT is the imaging modality of choice in both acute and CMI, allowing accurate diagnosis of the site and vessel affected, as well as some resultant complications. It can also exclude other differential diagnoses. ${ }^{4} 14$ A recent meta-analysis of the use of contrast-enhanced CT in occlusive mesenteric ischaemia demonstrated a pooled sensitivity of $93 \%$ and specificity of $96 \% .^{15}$ CT is widely available, and data acquisition is rapid.

Modern third-generation CT scanners have very high resolution $(\sim 0.5 \mathrm{~mm})$, and the image data can be manipulated and assessed in multiple planes, which is particularly useful for subtle stenoses and emboli. ${ }^{14}$ Sagittal reformatted images allow excellent visualisation of the vessel ostia as they arise from the abdominal aorta and can be used to grade vessel stenoses. Coronal reformatted images can demonstrate the entire vascular territory, particularly the distal branches and collateral vessels which can be difficult to visualise in the axial plane. ${ }^{16}$

Three-dimensional imaging is an interesting area for future development and is being incorporated into modern imaging software. ${ }^{17}$ Three-dimensional rendering can significantly 
increase detection of arterial occlusions in comparison with two-dimensional axial imaging. ${ }^{14} 18$

CT angiography (CTA) using iodinated intravenous contrast medium, has replaced catheter angiography as the first-line diagnostic tool. It is non-invasive and rapid. ${ }^{16}$ There is a risk of contrast-induced nephropathy in this patient group who are likely to have atherosclerotic disease elsewhere. ${ }^{5}$ However, the advantage of early accurate diagnosis of AMI potentially outweighs the disadvantages. Consideration of intravenous hydration, both prior to and following imaging, should be given to minimise any deleterious effects of the contrast medium. A recent study from Sweden showed that the mortality rate in patients undergoing contrast-enhanced CT was $42 \%$ vs $90 \%$ in the non-enhanced CT group. ${ }^{19}$ The same group demonstrated that although intravenous contrast did cause biochemical worsening of renal function, no patients required dialysis, and there was no mortality related to renal failure. ${ }^{20}$

Arterial thrombus may be visible as focal stenosis of the vessel or when calcified. Emboli are seen as filling defects within the lumen with a contrast 'cut-off' distal to the site of occlusion (figure 3). ${ }^{14}$ Venous thrombus, again, is visualised as a filling defect (figure 4). Other CT findings that should be looked for to suggest mesenteric ischaemia are bowel wall thickening ( $>3 \mathrm{~mm}$, but often $>8 \mathrm{~mm}$, particularly in venous thrombosis) and bowel dilatation, although these are not specific for mesenteric ischaemia. ${ }^{14}$ Bowel wall thinning is usually a sign of imminent perforation.

Pneumatosis and hepatic portal venous gas (figure 5) are seen in up to $30 \%$ and $14 \%$, respectively, of patients with mesenteric ischaemia, suggesting bowel perforation and the need for urgent surgery. ${ }^{14}{ }^{21}$ Neither of these signs are specific for mesenteric ischaemia, and they are being seen increasingly frequently in non-ischaemic conditions due to high-resolution imaging. ${ }^{22}$

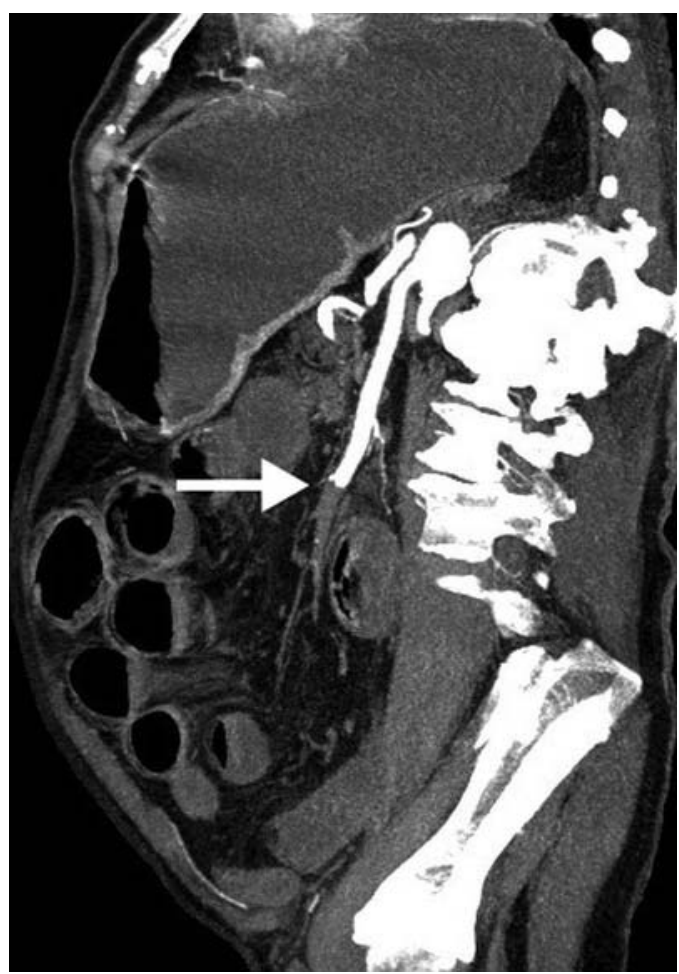

Figure $3 \mathrm{CT}$ angiogram demonstrating embolic disease within the mid-superior mesenteric artery with 'cut-off' of intra-arterial contrast medium at the site of luminal occlusion (arrow).

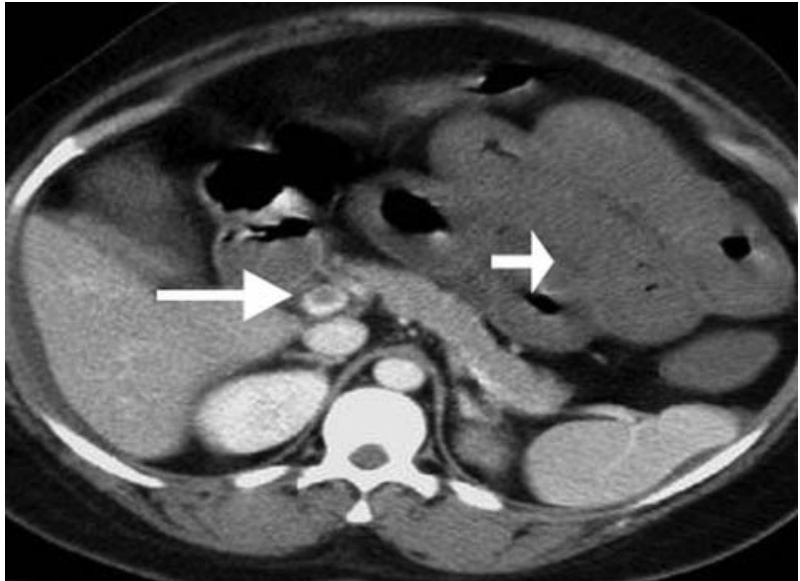

Figure 4 Thrombus within the superior mesenteric vein (long arrow) resulting small-bowel ischaemia depicted by thickened small-bowel loops with poor enhancement (short arrow) demonstrated at portal phase CT imaging.

Common non-ischaemic causes are inflammatory bowel disease, pancreatitis, abscesses and iatrogenic (eg, post-endoscopic retrograde cholangiopancreatography). ${ }^{21}$ Differential enhancement of bowel loops between arterial phases suggests ischaemic bowel segments, while lack of enhancement suggests bowel infarction. ${ }^{14}$ The majority of patients with SMA thromboembolic disease will have further synchronous emboli to other solid organs, which may be sought. Similarly, the source may be visible within the heart.

CTA is useful in the diagnosis of CMI, however, the confident diagnosis requires elimination of other abdominal pathologies. ${ }^{3}{ }^{14}$ Atherosclerosis is the commonest cause of CMI, and is very commonly seen on CTA in the general elderly population. ${ }^{3}$ The history may give clues to the diagnosis (abdominal angina or unexplained weight loss), as well as the presence of collateral mesenteric vessels. ${ }^{3}$ Sequelae within the bowel may be visible as

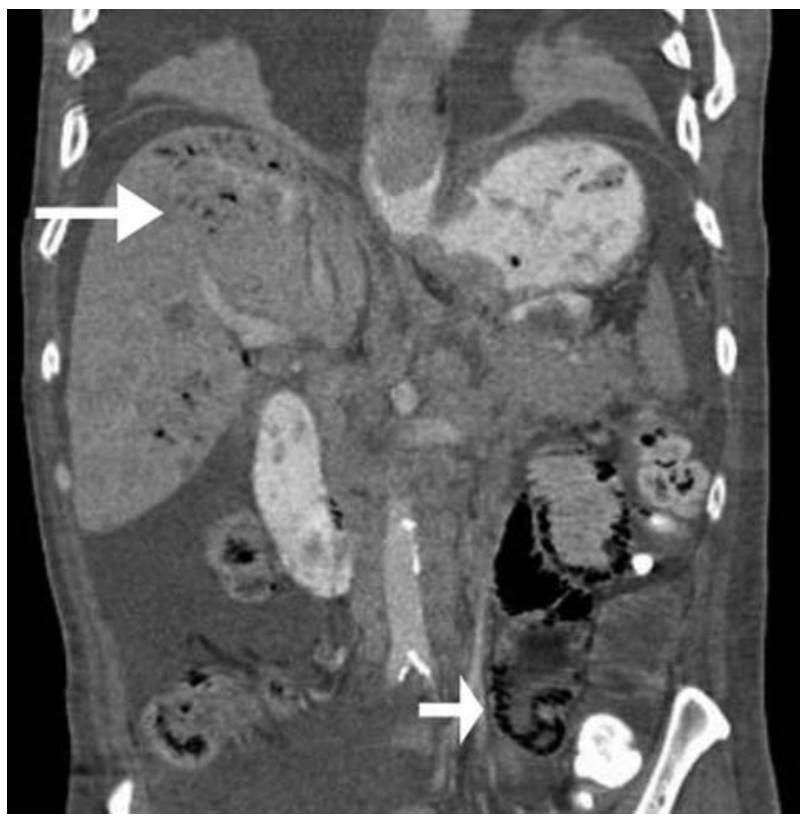

Figure 5 Coronal reformatted image from a portal phase CT study demonstrating gas locules within the portal vein (long arrow) and small-bowel ischaemia with poor wall enhancement and pneumatosis (short arrow). 


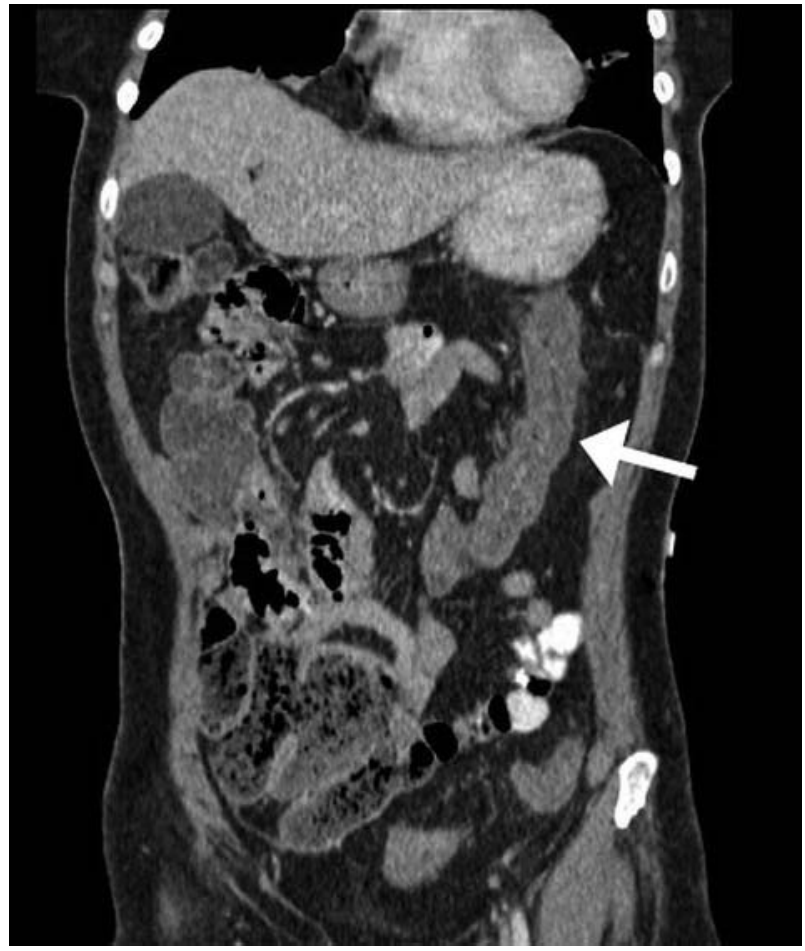

Figure 6 Thickened left hemicolon/colitis (arrow) in the distribution of the inferior mesenteric artery in a patient with aortic atherosclerosis. Ischaemic colitis was confirmed at endoscopy.

colitis (figure 6), or not be apparent. There may be ischaemic strictures, most commonly in the watershed zone between the SMA and IMA territories. Appearances of vasculitis may be difficult to differentiate from those of atherosclerotic disease, and may be represented by vascular wall thickening, occlusion, aneurysm formation and wall enhancement. ${ }^{1423}$

\section{Magnetic resonance angiography}

Contrast-enhanced magnetic resonance angiography (MRA) is generally not employed in AMI, due to both limited access and lengthy acquisition times in acutely unwell patients. ${ }^{4}$ However, MRA has been demonstrated to have comparable accuracy in the detection of both arterial thromboembolic disease and venous thrombus in the setting of CMI. ${ }^{23}$ Currently, the better spatial and temporal resolution of CTA allows better depiction of smaller vessels such as the narrower-calibre IMA, as well as the other abdominal viscera for resultant complications. ${ }^{23}$

Several MRA techniques are used according to the information required, and the clinical status of the patient. Non-intravenous contrast-medium techniques (time-of-flight and phase-contrast imaging) can be used in those with reduced glomerular filtration rates (GFR $<45 \mathrm{ml} / \mathrm{min}$ ). However, similar to $\mathrm{CT}$, the use of intravenous contrast-medium techniques demonstrate greater accuracy. ${ }^{23}$

\section{Management of mesenteric ischaemia}

The gold standard treatment of mesenteric ischaemia is revascularisation of the affected bowel to restore normal perfusion. Traditionally, the only treatment option was open surgery (embolectomy, endarteretomy and bypass grafting). ${ }^{4}$ Unfortunately, patients with mesenteric ischaemia are often elderly with multiple comorbidities and poor nutritional status. Consequently, endovascular treatment is an appealing minimally invasive option that has been used successfully since the late 1970s, and continues to be developed. ${ }^{4} 610$
In AMI, the patient requires aggressive emergency treatment to reperfuse ischaemic bowel and, ideally, prevent infarction. Early involvement of a vascular surgeon, interventional radiologist and intensivist is essential to guide resuscitation and decide on future treatment. The patient requires general anaesthetic and transfer to, ideally, a hybrid operating room with capability for both endovascular and open surgery. ${ }^{10}$

If the patient presents rapidly to hospital, has no signs of peritonism and a suspected or proven embolus/thrombus on CTA, then endovascular thrombectomy with or without thrombolysis can be attempted. Unfortunately, many studies have shown that this is suitable only in the minority of patients with AMI, since most have signs of bowel infarction and require laparotomy. ${ }^{6}$ Surgical techniques and endovascular techniques are described elsewhere in detail. 61024

The endovascular technique is performed via a standard femoral or brachial arterial puncture. A catheter is advanced to the occluded target artery (usually SMA), having being guided by the CTA. A brachial approach is helpful for cannulating the SMA, particularly when occluded due to its acute take-off angle from the aorta. ${ }^{1025}$ A confirmatory angiogram confirms the site of vessel occlusion, and the catheter is advanced as close as possible to the occlusion. A high-dose thrombolytic infusion is then started and continued for up to $48 \mathrm{~h}$, or until complete clot lysis, whichever is sooner. ${ }^{6}$ The patient requires careful observation for signs of peritonism or clinical deterioration during this period, in which case laparotomy is indicated.

A retrospective review of 48 patients with acute SMA occlusion showed complete clinical success in 63\% of patients with thrombolysis alone. ${ }^{6}$ Although not ideal, this may offer an option for very elderly patients or those with equivocal clinical signs, obviating the need for emergency laparotomy. This can be combined with balloon angioplasty and primary stenting, although results of stenting in AMI can be disappointing. ${ }^{4}$ Stenting is required, as the durability with angioplasty alone is poor. ${ }^{10}$ A retrospective review of the Swedish vascular registry suggested that endovascular treatment has better 30-day and 1-year mortality rates than open surgery for AMI, although these findings are limited by the fact that sicker patients would go directly to surgery, and that endovascular failures are treated surgically. ${ }^{12}$

If there are signs of peritonitis, clinically, or perforation on CT, then laparotomy is required to manually evaluate the whole length of bowel to assess viability. This cannot be achieved laparoscopically. ${ }^{4}$ Similarly, sometimes it is not possible to cannulate vessels endovascularly. At laparotomy, clearly infarcted areas of bowel can be resected, but potentially viable bowel should be left for approximately $30 \mathrm{~min}$, once perfusion has been established, or reassessed at a later planned laparotomy. ${ }^{4}$ Recently, a hybrid interventional radiology-surgery procedure has been described which combines the benefits of both endovascular stenting with access directly to the site of occlusion and assessment of bowel viability. ${ }^{24}$ Results of the retrograde open mesenteric stenting procedure in a small group of AMI patients, showed an in-hospital mortality rate of $17 \%$, compared with $>80 \%$ in the surgical bypass patients, and $100 \%$ in percutaneously stented patients. ${ }^{24}$ In AMI, usually only the affected single vessel is bypassed/stented to minimise procedure time and stress in an unwell patient. ${ }^{24}$

Percutaneous angioplasty and stenting (PTAS) has evolved to become the first-line treatment for CMI (figure 7A-D), although clinically fit patients should be offered open revascularisation. ${ }^{25-27}$ PTAS has significantly better short-term morbidity and mortality than open surgery, where primary vessel patency and absence of patient symptoms were both around $62 \%$ at 36 months. ${ }^{25}$ However, there were no deaths from CMI or AMI in this period. 


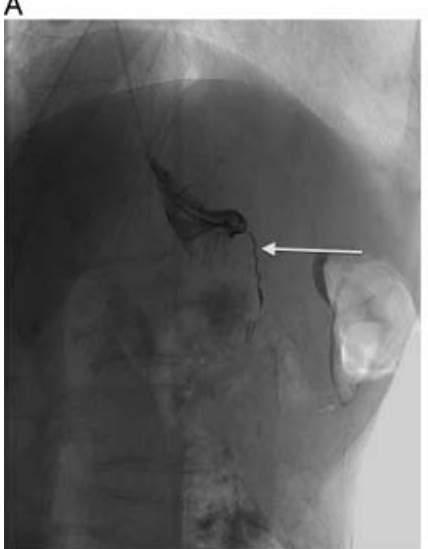

B

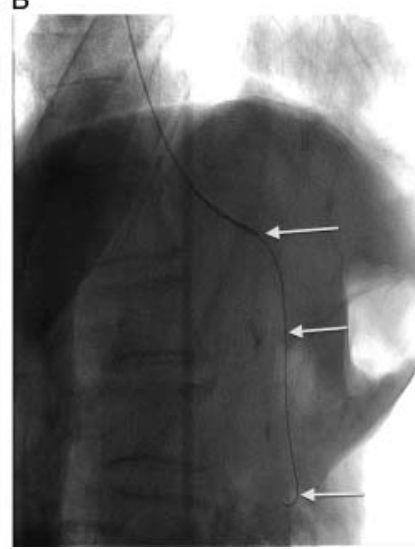

C

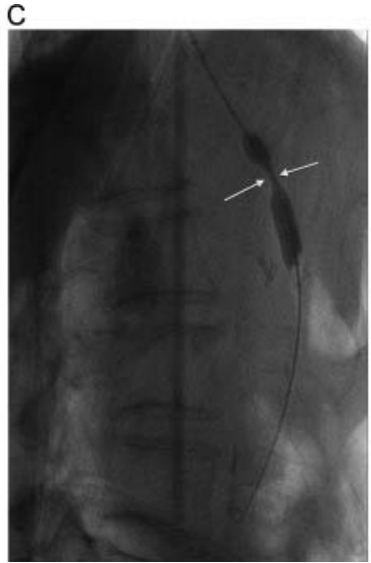

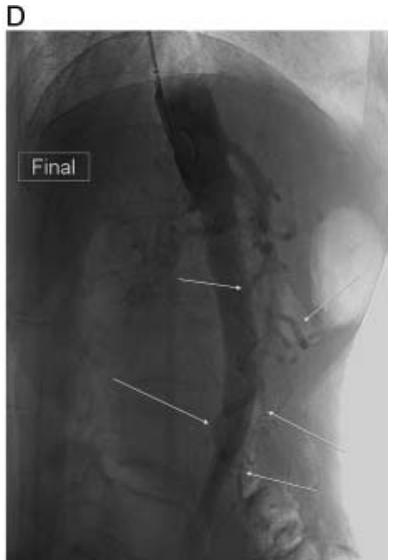

Figure 7 (a) Initial oblique superior mesenteric artery (SMA) catheter angiogram in a 72-year-old man with confirmed chronic mesenteric ischaemia. The angiogram shows almost total proximal SMA occlusion with only a trickle of contrast passing distally. Note, brachial (superior) approach of catheter for easier cannulation of the SMA. (b) A hydrophilic guide wire is passed through the SMA occlusion and distal control is secured. (c) An angioplasty balloon is railroaded over the guide wire and inflated for $60 \mathrm{~s}$. Note, waisting of the balloon at the point of maximum stenosis. (d) Completion oblique SMA angiogram after successful angioplasty and insertion of a balloon-expanded covered stent. Note, good distal contrast run-off compared with figure 7a.

This data suggests that a significant number of patients will require further radiological follow-up and interventional radiology procedures such as angioplasty of stent stenoses. However, it also allows time for patient optimisation (such as regaining normal body weight) if elective surgery is indicated. Stenting of multiple mesenteric arteries increased the duration of stent patency, and can be attempted. $^{25}$

\section{Main messages}

- Atherosclerotic changes in the mesenteric arteries are common in the elderly, although acute mesenteric ischaemia (AMI) is uncommon. A high suspicion of mesenteric ischaemia coupled with a careful clinical history is required to allow prompt diagnosis.

- AMl is a vascular surgical emergency. Delays in diagnosis lead to greater bowel ischaemia and infarction, with associated increased morbidity and mortality. Early involvement of a vascular surgeon and interventional radiologist is essential.

- Multiple collaterals pathways exist and can develop between the three main arteries providing mesenteric arterial supply. Knowledge of these arterial territories is useful to understand the likely affected viscera (end organs), and planning endovascular treatment.

- Catheter angiography has been superseded by CT angiography (CTA) in the diagnosis of mesenteric ischaemia. CTA is readily available and provides high sensitivity and specificity in the diagnosis of AMI. Chronic mesenteric ischaemia is often a diagnosis of exclusion, but should be remembered in elderly patients with visceral atherosclerosis and unexplained weight loss for which no other cause is found.

- Hepatic portal venous gas on abdominal x-ray and CT is an ominous finding in the context of mesenteric ischaemia, however, there are multiple non-ischaemic causes. Active discussion between specialities is encouraged, particularly, correlating history and clinical findings with the radiological findings.

- Endovascular stenting offers an effective minimally invasive treatment for mesenteric ischaemia, with good short-term outcomes.

\section{Current research questions}

- Can improvements in CT technology and software be used to assess bowel viability and prevent the need for exploratory laparotomy?

- How can we identify the patient population at risk with chronic mesenteric ischaemia from the background general population with mesenteric atherosclerosis?

- Is there a role for treating asymptomatic patients with moderate/severe multivessel mesenteric atherosclerosis to prevent the progression to acute mesenteric ischaemia?

- Will the use of covered stents reduce the rate of recurrent stenoses in patients treated endovascularly?

\section{Summary}

Acute mesenteric ischaemia (AMI) is a vascular emergency, which requires immediate investigation and treatment to prevent bowel infarction. Chronic mesenteric ischaemia (CMI) should be considered in elderly patients with unexplained weight loss and signs of mesenteric atherosclerosis or the typical 'mesenteric angina' history.

- Contrast-enhanced CT is widely available and accessible, and provides rapid and effective diagnosis of both $\mathrm{AMI}$ and CMI. It should be considered as the first-choice diagnostic method.

- Early vascular surgery and interventional radiology input is required for determining the most suitable treatment option for those elderly patients who may be critically unwell or malnourished. Endovascular treatment continues to develop both as a hybrid procedure with open surgery or as a stand-alone procedure. 


\section{Key references}

- Acosta S. Epidemiology of mesenteric vascular disease: clinical implication. Semin Vasc Surg 2010;23:4-8.

- Menke J. Diagnostic accuracy of multidetector CT in acute mesenteric ischaemia:systematic review and meta-analysis. Radiology 2010;256:93-101.

- Cognet F, Salem DB, Dranssart M, et al. Chronic mesenteric ischaemia: imaging and percutaenous treatment. Radiographics 2002;22:863-80.

- Resch TA, Acosta S, Sonesson B. Endovascular techniques in acute arterial mesenteric ischaemia. Semin Vasc Surg 2010;23:29-35.

- Peck MA, Conrad MF, Kwolek CJ, et al. Intermediate-term outcomes of endovascular treatment for symptomatic chronic mesenteric ischaemia. J Vasc Surg 2010;51:140-7. e1-2.

\section{MULTIPLE CHOICE QUESTIONS (TRUE (T)/FALSE (F); ANSWERS AFTER THE REFERENCES)}

1. Regarding mesenteric vascular anatomy:

A. The CA supplies the mid-gut

B. The SMA give rise to the middle colic artery

C. The SMA supplies the left hemicolon

D. The arc of Riolan provides collaterals between SMA and IMA territories

E. The SMA has an acute angle of origin with the aorta

2. Regarding aetiology and clinical presentation of mesenteric ischaemia:

A. Acute mesenteric ischaemia may present with nonspecific symptoms

B. Chronic mesenteric ischaemia can present with postprandial pain

C. Raised lactate levels are 100\% specific for acute mesenteric ischaemia

D. Chronic mesenteric ischaemia is most commonly due to vasculitis

E. Venous occlusion does not result in acute mesenteric ischaemia

3. Regarding radiological investigation of mesenteric ischaemia:

A. MRI is the first imaging modality of choice in acute mesenteric ischaemia

B. Plain film imaging is highly sensitive in patients with acute abdominal pain

C. CTA has superseded catheter angiography in the diagnosis of mesenteric ischaemia

D. US has no role in the evaluation of mesenteric ischaemia

4. Regarding CT evaluation of mesenteric ischaemia:

A. CT has low sensitivity in the demonstration of arterial emboli

B. Pneumatosis coli is an imaging feature of acute ischaemia

C. Chronic mesenteric ischaemia cannot be identified at CT

D. Contrast-induced nephropathy is not a risk in this group of patients

E. Multidetector CT allows depiction of small vessels due to its high resolution

5. Regarding management of mesenteric ischaemia:

A. There is no role for endovascular therapy in the management of acute mesenteric ischaemia
B. There is no role for endovascular therapy in the management of chronic mesenteric ischaemia

C. Endovascular therapy has a lower morbidity rate than surgery

D. Symptoms are more likely to recur in those undergoing surgery

Acknowledgements The opinions expressed by JJH are his own and not necessarily those of the Ministry of Defence. We thank the NIHR Cambridge Biomedical Research Centre for their support.

Contributors $\mathrm{JJH}$ wrote the revised manuscript and supplied the IR figures. SU wrote the initial manuscript and supplied figures 1-6. JJH, SU, RU and AG all helped edit the final article for submission. JJH and SU are the guarantors for the article. AG is the corresponding author.

Funding None.

Competing interests None.

Provenance and peer review Commissioned; externally peer reviewed.

\section{REFERENCES}

1 Oldenburg WA, Lau LL, Rodenberg TJ, et al. Acute mesenteric ischemia: a clinical review. Arch Intern Med 2004;164:1054-62.

2 Acosta S. Epidemiology of mesenteric vascular disease: clinical implications. Semin Vasc Surg 2010;23:4-8.

3 Cognet F, Ben Salem D, Dranssart M, et al. Chronic mesenteric ischemia: imaging and percutaneous treatment. Radiographics 2002;22:863-79; discussion 879-80.

4 Wyers MC. Acute mesenteric ischemia: diagnostic approach and surgical treatment. Semin Vasc Surg 2010;23:9-20.

5 Valentine RJ, Martin JD, Myers SI, et al. Asymptomatic celiac and superior mesenteric artery stenoses are more prevalent among patients with unsuspected renal artery stenoses. J Vasc Surg 1991:14:195-9.

6 Schoots IG, Levi MM, Reekers JA, et al. Thrombolytic therapy for acute superior mesenteric artery occlusion. J Vasc Interv Radiol 2005:6:317-29.

7 Endean ED, Barnes SL, Kwolek CJ, et al. Surgical management of thrombotic acute intestinal ischemia. Ann Surg 2001;233:801-8.

8 Acosta S, Ogren M, Sternby NH, et al. Clinical implications for the management of acute thromboembolic occlusion of the superior mesenteric artery: autopsy findings in 213 patients. Ann Surg 2005;241:516-22

9 Walker TG. Mesenteric vasculature and collateral pathways. Semin Intervent Radiol 2009:26:167-74.

10 Resch TA, Acosta S, Sonesson B. Endovascular techniques in acute arterial mesenteric ischemia. Semin Vasc Surg 2010;23:29-35.

11 Acosta S, Nilsson TK, Bjorck M. D-dimer testing in patients with suspected acute thromboembolic occlusion of the superior mesenteric artery. Br J Surg 2004;91:991-4

12 Block TA, Acosta S, Bjorck M. Endovascular and open surgery for acute occlusion of the superior mesenteric artery. J Vasc Surg 2010;52:959-66.

13 Bradbury MS, Kavanagh PV, Bechtold RE, et al. Mesenteric venous thrombosis: diagnosis and noninvasive imaging. Radiographics 2002;22:527-41.

14 Horton KM, Fishman EK. Multidetector CT angiography in the diagnosis of mesenteric ischemia. Radiol Clin North Am 2007:45:275-88.

15 Menke J. Diagnostic accuracy of multidetector CT in acute mesenteric ischemia: systematic review and meta-analysis. Radiology 2010;256:93-101.

16 Horton KM, Fishman EK. Multi-detector row CT of mesenteric ischemia: can it be done? Radiographics 2001:21:1463-73.

17 Horton KM, Fishman EK. Volume-rendered 3D CT of the mesenteric vasculature: normal anatomy, anatomic variants, and pathologic conditions. Radiographics 2002;22:161-72

18 Chen JK, Johnson PT, Horton KM, et al. Unsuspected mesenteric arterial abnormality: comparison of MDCT axial sections to interactive 3D rendering. AJR Am J Roentgenol 2007;189:807-13.

19 Wadman M, Block T, Ekberg O, et al. Impact of MDCT with intravenous contrast on the survival in patients with acute superior mesenteric artery occlusion. Emerg Radiol 2010;17:171-8.

20 Acosta S, Bjornsson S, Ekberg 0, et al. CT angiography followed by endovascular intervention for acute superior mesenteric artery occlusion does not increase risk of contrast-induced renal failure. Eur J Vasc Endovasc Surg 2010;39:726-30.

21 Nelson AL, Millington TM, Sahani $D$, et al. Hepatic portal venous gas: the ABCs of management. Arch Surg 2009;144:575-81; discussion 581.

22 Hou SK, Chern CH, How CK, et al. Hepatic portal venous gas: clinical significance of computed tomography findings. Am J Emerg Med 2004;22:214-18.

23 Shih MC, Hagspiel KD. CTA and MRA in mesenteric ischemia: part 1, role in diagnosis and differential diagnosis. AJR Am J Roentgenol 2007;188:452-61.

24 Wyers MC, Powell RJ, Nolan BW, et al. Retrograde mesenteric stenting during laparotomy for acute occlusive mesenteric ischemia. J Vasc Surg 2007:45:269-75. 
25 Peck MA, Conrad MF, Kwolek CJ, et al. Intermediate-term outcomes of endovascular treatment for symptomatic chronic mesenteric ischemia. J Vasc Surg 2010:51:140-7. e1-2.

26 Fioole B, van de Rest HJ, Meijer JR, et al. Percutaneous transluminal angioplasty and stenting as first-choice treatment in patients with chronic mesenteric ischemia. J Vasc Surg 2010;51:386-91.

27 Atkins MD, Kwolek CJ, LaMuraglia GM, et al. Surgical revascularization versus endovascular therapy for chronic mesenteric ischemia: a comparative experience. J Vasc Surg 2007;45:1162-71.

\section{ANSWERS}

1. (A) $\mathrm{F} ;(\mathrm{B}) \mathrm{T} ;(\mathrm{C}) \mathrm{F} ;(\mathrm{D}) \mathrm{T}$; (E) $\mathrm{T}$

2. (A) $T_{;}$(B) $T_{;}$(C) $F$; (D) $F$; (E) $F$

3. (A) $\mathrm{F} ;(\mathrm{B}) \mathrm{F}$; (C) $\mathrm{T}$; (D) $\mathrm{F}$

4. (A) $\mathrm{F}$; (B) $\mathrm{T}$; (C) $\mathrm{F}$; (D) $\mathrm{F}$; (E) $\mathrm{T}$

5. (A) $\mathrm{F}$; (B) $\mathrm{F}$; (C) $\mathrm{T}$; (D) F 